\title{
CORRIGENDUM
}

\section{Corrigendum for: ICE1 of Poncirus trifoliata functions in cold tolerance by modulating polyamine levels by interacting with arginine decarboxylase}

\section{Xiao-San Huang ${ }^{1}$, Qinghua Zhang ${ }^{1}$, Dexin Zhu' ${ }^{1}$, Xingzheng Fu' ${ }^{1}$, Min Wang ${ }^{1}$, Qian Zhang ${ }^{1}$, Takaya Moriguchi ${ }^{2}$ and Ji-Hong Liu ${ }^{1, *}$}

${ }^{1}$ Key Laboratory of Horticultural Plant Biology (MOE), College of Horticulture and Forestry Science, Huazhong Agricultural University, Wuhan 430070, PR China

${ }^{2}$ National Institute of Fruit Tree Science, Tsukuba, 305-8605, Japan

* To whom correspondence should be addressed. E-mail: liujihong@mail.hzau.edu.cn

Journal of Experimental Botany, Vol. 66, No. 11, pp. 3259-3274, 2015

The authors of the above article would like to correct the acknowledgements as follows:

This work was financially supported by the National Science Foundation of China (31272147, 31320103908), the Ministry of Agriculture, and the National High Technology Research and Development Program of China (2011AA100205).

The project number '31272147, 31320103908' was originally incorrectly given as '31272147, 20130146110019'. 